\title{
El desarrollo del liderazgo a través de un congreso organizado por estudiantes. Estudio pre-post intervención*
}

\author{
M Ángeles de Juan Pardo ${ }^{a, b}$, Pilar Fuster-Linares ${ }^{\text {a,c }}$, Alberto Gallart Fernández-Puebla ${ }^{\text {a,d }}$, Encarna \\ Rodriguez $^{\mathrm{a}, \mathrm{e}}$, Laia Wennberg ${ }^{\mathrm{a}, \mathrm{f}}$ y Maria Luisa Martín-Ferreres ${ }^{\mathrm{a}, \mathrm{g}}$ \\ aDepartamento de Enfermería. Facultad de Medicina y Ciencias de la Salud. Universitat Internacional de Catalunya.

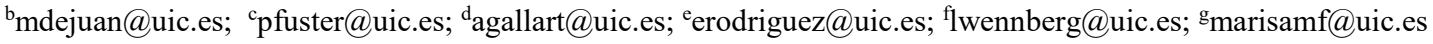

\begin{abstract}
A Student Led Conference was planned and organized by nursing students enrolled in two elective modules (Interdisciplinary Care of Older Adults and Old Age and Dependency) across two academic years (201819 and 2019-20). Students participated actively in the conference, with oral communications (as part of their course assignment), or moderating a round table. The results shown a significative increase of the level of leadership in the students that led the conference, and a high level of satisfaction in the participants in the conference. This innovative teaching method, may be of interest and could be applied in different contexts, as it is necessary to implement methodologies that improve competence development as leadership and communication.
\end{abstract}

Keywords: student-led conferences; leadership; nursing; team work; emotional intelligence; critical thinking; communication skills.

\section{Resumen}

Presentamos una Student Led Conference planificada y organizada por estudiantes de enfermería matriculados en dos asignaturas optativas (Abordaje Interdisciplinar al Anciano y Ancianidad y Dependencia). Los estudiantes también participaron activamente en el congreso, presentando comunicaciones orales (como parte de la evaluación de la asignatura), o moderando una mesa redonda. Los resultados muestran un incremento significativo del nivel de liderazgo en los estudiantes que organizaron el congreso, así como un alto nivel de satisfacción en los participantes en el mismo. Este método docente innovador, puede ser de interés y aplicado en numerosos ámbitos, ya que es necesario implementar metodologías que ayuden a desarrollar competencias como liderazgo y comunicación.

\footnotetext{
* Proyecto que recibió el Segundo premio de la Beca Puig de Innovación docente. 25/05/2018-30/06/2020. Los resultados de este Proyecto se han publicado en la revista Nurse Education Today. De Juan Pardo, M.A., Fuster, P., Gallart, A., Rodríguez, E., Wennberg, L., Martín-Ferreres M.L. Fostering leadership competence and satisfaction in nursing undergraduates through student-led conference: A quasi-experimental pre-post study. Nurse Educ Today. 2021 Mar;98:104748. doi: 10.1016/j.nedt.2021.104748. Epub 2021 Jan 18. Las tablas 1 y 2 están extraidas de dicho artículo. En esta comunicación se destacan los aspectos de innovación docente y los resultados de comunicación y liderazgo que pueden ser extrapolables a cualquier área de conocimiento.
} 
Palabras clave: Student Led Conferences; liderazgo; enfermería; trabajo en equipo; inteligencia emocional; pensamiento crítico; habilidades de comunicación.

\section{Introducción}

Debido al contexto de gran complejidad que existe actualmente en el ámbito sanitario, todavía más patente en las situaciones de pandemia, como la que se está viviendo en la actualidad con el COVID-19, los gestores de Enfermería, destacan la necesidad de que los profesionales de Enfermería incorporen la competencia de liderazgo en su práctica diaria (Estalella, 2020; Shingler-Nace, 2020). Este liderazgo clínico se considera necesario entre los profesionales de enfermería para proporcionar cuidados seguros y de calidad en un sistema sanitario, cada vez más complejo, integrado por pacientes frágiles, pluripatológicos, y en su mayoría de edad avanzada (Glazer et al., 2011).

La American Association Colleges of Nursing (AACN, 2008), coincide en identificar la competencia de liderazgo como uno de los elementos clave de los profesionales de enfermería. Para ello, al igual que otros autores, considera relevante que se trabaje y desarrolle esta competencia durante los estudios universitarios (Galuska, 2015; Ward et al., 2016).

En esta línea, se han desarrollado diversas metodologías con la finalidad de impulsar el desarrollo de la competencia de liderazgo entre los estudiantes de Enfermería. Entre estas metodologías docentes, se encuentran las denominadas Student-Led Conferences (Galuska, 2015; Ward, 2016) como actividades innovadoras, que consisten en la organización de conferencias lideradas por estudiantes y en nuestro caso además, integradas dentro de una asignatura curricular. Sin embargo, no se han encontrado estudios que comprueben la eficacia en el incremento del nivel de liderazgo adquirido a través de esta metodología.

Por este motivo, y debido a la necesidad de mantener los conocimientos actualizados en el área de geriatría de los estudiantes en Enfermería, incrementar su motivación sobre ese tema e incrementar su liderazgo, se decidió modificar dos asignaturas de $4^{\circ}$ de Grado de la Universitat Internacional de Catalunya (UIC), dándoles el formato de jornada científica.

Este hecho supuso transformar totalmente las asignaturas, a través de una innovación disruptiva (Piolineta, 2017). Con esta nueva metodología los alumnos tienen la oportunidad de experimentar el contexto real de la organización y gestión de un evento científico para desarrollar las competencias de liderazgo y comunicación de resultados. 


\section{Objetivos}

El objetivo principal del estudio fue evaluar el impacto de un student-led conference sobre el nivel de liderazgo de los estudiantes del grado de Enfermería, así como el grado de satisfacción de los participantes en el evento.

\section{Desarrollo de la innovación}

Como parte del programa de dos asignaturas optativas de $4^{\circ}$ de grado de Enfermería de la UIC, Abordaje Interdisciplinar al Anciano y Ancianidad y Dependencia, se incorporó la organización y desarrollo de una jornada científica liderada por estudiantes.

El cronograma de la asignatura se planificó siguiendo las fases de organización del congreso, de manera que gradualmente el alumno pudiera ir aprendiendo e implementando las gestiones de organización.

Para fomentar la capacitación en liderazgo y gestión, todos los alumnos formaron parte del comité científico o del organizador, asumiendo las funciones de elegir el tema del congreso, seleccionar y contactar con ponentes expertos en el área, elaborar el programa de la jornada, conseguir financiación (materiales y coffee break), reserva de aula, gestión de las inscripciones y certificados. Además, formó parte de su trabajo la atención de los ponentes y asistentes.

En relación a la adquisición de la competencia de comunicación, todos los alumnos participaron activamente en el contacto con profesionales, sponsors o ponentes invitados y los demás asistentes al acto. Durante el evento, o bien presentaron las comunicaciones orales, o bien, fueron autores de las mismas. Además, algunos fueron moderadores de mesas redondas o intervienieron en la apertura o clausura del acto. Para desarrollar estas tareas, los estudiantes fueron guiados y supervisados por la coordinadora de la asignatura durante el periodo lectivo. El evento científico culminó la asignatura.

Para medir el nivel de liderazgo de los estudiantes organizadores de la Jornada, se utilizó la escala ES-SALI (Fuster, 2021). Es una escala validada al castellano, de la escala original (Smola, 2003, 1988), que mide las percepciones de los estudiantes de enfermería sobre el comportamiento de liderazgo, en sus cuatro dimensiones: pensamiento estratégico (de 0-44); inteligencia emocional (de 0-32); impacto e influencia (de 0-28) y trabajo en equipo (de 0-56). Está compuesta de 40 items, tipo Lickert 0-4, siendo 0 "normalmente no me comporto así” y 4 "casi siempre me comporto asín”, con una puntuación total de 0 a 160.

Para medir el nivel de satisfacción de los participantes en el congreso, se utilizó un cuestionario autoadministrado de elaboración propia. Incluye preguntas tipo Likert del 1 al 10 (siendo 1 nada satisfecho y 10 totalmente satisfecho), con valores de 5-50, y preguntas abiertas sobre su percepción del congreso, propuestas de mejora, temas propuestos para futuras ediciones.

El primer día de las asignaturas mencionadas, se explicó brevemente en qué consistiría el evento que iban a organizar y se administró el cuestionario ES-SALI. Posteriormente, se formaron los equipos (comité científico y comité organizador). Cada equipo eligió a su presidente, quienes se reunirían semanalmente 
con la coordinadora de la asignatura, y después con sus respetivos equipos. También escogieron a los moderadores para la mesa redonda de ponentes, y la/s mesa/s de comunicaciones. Además del presidente del comité científico y del organizador, que ya iban a exponer en la jornada, los demás, tuvieron que presentar las comunicaciones. Todos los alumnos matriculados fueron también autores de una de las comunicaciones orales. El tema de la jornada se eligió entre los propuestos por los participantes en la edición anterior, u otros que aportaron los alumnos actualmente matriculados. Por la situación actual de envejecimiento de la población, la temática suele ser de gran actualidad y relevancia para la práctica profesional. Finalmente, se concretó la fecha, la sala y el horario de la jornada. El programa siguió la siguiente estructura: mesa de apertura, mesa redonda (con ponentes invitados), pausa café, mesas de comunicaciones, lectura de conclusiones, entrega de premios (premio a la mejor comunicación), y clausura.

El segundo día de clase, también fue clave para la organización del evento. El comité científico propuso posibles ponentes (del ámbito profesional), para la mesa redonda. En este punto fueron asesorados por la propia coordinadora de la asignatura y otros docentes del grado. Se acordó quién inauguraría la jornada (como en ocasiones anteriores, se invitó a la directora del Departamento de Enfermería), y quién la clausuraría. La coordinación entre comité científico y organizador sería clave para: mantener coherencia entre las comunicaciones sobre el tema escogido y evitar repeticiones o redundancias entre las intervenciones durante la jornada.

A lo largo de la asignatura se programaron tutorías grupales, con la finalidad de dirigirles e impulsarles en las tareas de organización, ayudan a abordar pequeños conflictos de equipo, o a resolver problemas cuando apareciesen. Se procuró que fuesen los propios estudiantes quienes los resolviesen, manteniendo la coordinadora de la asignatura un papel de mediadora y de guía, destacando de nuevo el liderazgo en la toma de decisiones que tienen los alumnos en esta metodología.

Otros dos momentos clave, ambos, el día de la jornada, fueron "la cuenta atrás", con la mañana de ensayos, y "remates" (envolver regalos para ponentes, preparar mesa para la pausa café, acreditaciones, aguas y carteles para los ponentes, etc.). Y en la propia jornada, “icada uno a sus puestos!”, en la que se pudo comprobar el resultado del trabajo en equipo, el cuidado de los detalles y la responsabilidad de cada uno de los miembros.

Al finalizar, los participantes en la jornada (organizadores y asistentes) cumplimentaron un cuestionario de satisfacción y evaluación de la misma, y los organizadores, volvieron a realizar el cuestionario de liderazgo ES-SALI (Fuster, 2021).

Se obtuvo la aprobación del Comité de Ética en la Investigación de la universidad donde se llevó a cabo el estudio. Todos los estudiantes que participaron en el estudio, firmaron el consentimiento informado, tras recibir información y asegurar su voluntariedad. Para preservar el anonimato, las encuestas fueron codificadas, y solo la investigadora principal tuvo acceso a los datos completos. 


\section{Resultados}

Mostramos aquí los resultados de las jornadas realizadas en los cursos académicos 2018-19 y 2019-20 con las temáticas "la comunicación con pacientes en situación terminal" y "el maltrato en el anciano". Un total de 31 estudiantes participaron en el proyecto (un 88.6\% matriculados en las asignaturas desde las que se organiza la jornada). Un total de 14 hombres y 17 mujeres, con una edad media de 25.7 (desviacion estandar 6.2), siendo la edad minima 21 años y la máxima 49. A las jornadas asistieron un total de 89 personas (35 estudiantes organizadores, 11 de otras asignaturas, 17 profesores, 15 ponentes y 8 profesionales).

Dando respuesta a los objetivos del estudio, se presentan resultados del impacto de las jornadas en el nivel de liderazgo de los estudiantes del grado de Enfermería matriculados en las asignaturas mecionadas, y el grado de satisfacción de los participantes en el evento.En cuanto al nivel de liderazgo adquirido por los estudiantes organizadores del evento antes y después del mismo (pre y post), los resultados del cuestionario ES_SALI muestran un aumento significativo $(\mathrm{p}<0,01)$ tanto de la puntuación total del cuestionario (nivel de liderazgo), , como de cada una de las dimensiones de la escala ES_SALI: pensamiento estratégico, inteligencia emocional, impacto e influencia y trabajo en equipo. El incremento del liderazgo de estos estudiantes medido antes y después de la jornada, fue superior al 9\%(Tabla 1). La dimensión en la que hubo el mayor incremento, fue la de impacto e influencia, con un aumento del 10,99\%.

Tabla 1. Resultados del nivel de liderazgo y sus dimensiones Pre-Post Congreso

\begin{tabular}{|c|c|c|c|}
\hline & $\begin{array}{l}\text { Resultados ES_SALI } \\
(n=31) . \text { Media (DE) }\end{array}$ & p Pre-Post & Cambio percentual \% \\
\hline Total ES_SALI Pre $\mathbf{T}$ & $121,31(18,278)$ & & $9.42 \%$ \\
\hline \multirow[t]{2}{*}{ Total ES_SALI Post } & $132,74(13,609)$ & $<0,001^{b}$ & \\
\hline & $\begin{array}{c}\text { Resultados según } \\
\text { dimensiones de ES_SALI }\end{array}$ & $\begin{array}{l}\text { p Pre-Post según } \\
\text { dimensiones }\end{array}$ & $\begin{array}{l}\text { Cambio percentual } \\
\text { según dimensiones }\end{array}$ \\
\hline $\begin{array}{l}\text { Pensamiento } \\
\text { estratégico Pre }\end{array}$ & $32,84(5,113)$ & $\mathbf{0 , 0 0 2 ^ { \mathrm { a } }}$ & $9.71 \%$ \\
\hline $\begin{array}{l}\text { Pensamiento } \\
\text { estratégico Post }\end{array}$ & $36,03(4,151)$ & & \\
\hline Inteligencia & $25,65(3,592)$ & & \\
\hline Emocional Pre $\Phi$ & & $\mathbf{0 , 0 0 3} 3^{b}$ & $8,93 \%$ \\
\hline $\begin{array}{l}\text { Inteligencia } \\
\text { Emocional Post }\end{array}$ & $27,94(2,620)$ & & \\
\hline Impacto e & $19,65(4,637)$ & & \\
\hline Influencia Pre & & $0,002^{\mathrm{a}}$ & $10,99 \%$ \\
\hline $\begin{array}{l}\text { Impacto e } \\
\text { Influencia Post }\end{array}$ & $21,81(3,146)$ & & \\
\hline Trabajo en & $43,19(7,382)$ & & \\
\hline
\end{tabular}




\begin{tabular}{l}
\hline Equipo Pre \\
Trabajo en \\
Equipo Post
\end{tabular}

Respecto a la satisfacción con la Jornada, los cuestionarios de satisfacción de los participantes muestran resultados muy satisfactorios. El 68'53\% de los participantes (61), cumplimentó el cuestionario de satisfacción. La satisfacción media de los asistentes a las jornadas fue de 9,33 puntos sobre 10 (Tabla 2).

Tabla 2. Resultados de los cuestionarios de satisfacción de la jornada

\begin{tabular}{cc}
\hline $\mathbf{N}=\mathbf{6 1}$ & Media (desviación Estándar) \\
\hline Satisfacción general con la jornada & $9,33(0,77)$ \\
Organización de la jornada & $9.43(0.86)$ \\
Duración de la Jornada & $8.85(1.21)$ \\
Atención por parte de la organización & $9.59(0.94)$ \\
Interés del contenido de la jornada & $9.59(0.69)$ \\
\hline
\end{tabular}

Todos los comentarios recogidos en las preguntas abiertas fueron positivos. A continuación se recogen algunos de los comentarios de los profesionales que asistieron a las Jornadas: "Os felicito por el alto nivel de contenido y también por la dedicación y compromiso que destilan. También os agradezco el hecho de poder participar en la clausura y animar a los profesionales del futuro a seguir con esta trayectoria iniciada". Un ponente invitado, que asistió a todo el Congreso: "Me ha sorprendido gratamente los temas tratados y su profundidad. La participación de todos los implicados en el congreso y la buena organización. Me voy muy satisfecho de haber asistido. He aprendido mucho de gente tan joven y preparada. Gracias por invitarnos". Los comentarios de los estudiantes de la organización también fueron muy positivos, como se puede ver en los siguientes ejemplos: "Me ha proporcionado muchas competencias tanto a nivel profesional como a nivel personal. He podido ver todo el trabajo que hay detrás de todas las jornadas y congresos. A nivel individual el trabajo en equipo, constancia, compromiso, etc."; "Me ha sido muy útil, no simplemente teóricas, sino que he aprendido a trabajar en equipo y ser capaz de participar en el congreso de forma activa". "Considero que hemos aprendido todo el gran trabajo que hay detrás de preparar una jornada y que muchas veces no se ve ni se piensa". 


\section{Conclusiones}

Se confirma que un Student-led Conference puede incrementar significativamente el nivel de liderazgo de los estudiantes que organizan dicho evento.

Es importante la planificación y guía constante del profesor, para el desarrollo de la organización, así como la resolución de problemas.

Son numerosos los beneficios aportados por este tipo de actividades. Entre ellos, destacan además del incremento de liderazgo, el desarrollo de habilidades como el trabajo en equipo o la comunicación y gestión de la organización, así como las personales.

La Student-Led Conference es una actividad innovadora que puede ser transferible a cualquier tipo de curriculum educativo y que tal y como nosotros la presentamos es sostenible.

\section{Referencias}

AACN (2008). The Essentials of Baccalaureate Education for Professional Nursing Practice. American Association of Colleges of Nursing. https://www.aacnnursing.org/portals/42/publications/baccessentials08.pdf

ESTALELLA, G.M., ZABALEGUI, A. y GUERRA, S.S. (2020). Gestión y liderazgo de los servicios de enfermería en el plan de emergencia de la pandemia Covid-19: La Experiencia Del Hospital Clínic De Barcelona. Enfermería Clínica. no 31, p. S12-S17. Doi:10.1016/j.enfcli.2020.05.002

FUSTER, P., MARTÍN-FERRERES, M., RODRIGUEZ, E., WENNBERG, L. y GALLART FERNÁNDEZ-PUEBLA, A. (2020). Dimensions of Leadership in Undergraduate Nursing Students. Validation of a Tool. Nurse Education Today. no 95: 104576. Doi: 10.1016/j.nedt.2020.104576. Epub 2020 Sep 3.

GALUSKA, L.A. (2015). Dedicated education units: Partnerships for building leadership competency. J. Nurse Education Today. n54, p. 385-388. Doi:10.3928/01484834-20150617-05

GLAZER, G., IVES ERICKSON, J., MYLOTT, L., MULREADY-SHICK, J. y BANISTER, G. (2011). Partnering and leadership: Core requirements for developing a dedicated education unit. Journal of Nursing Administration $\mathrm{n}^{\circ}$ 41, p. 401-406. Doi:10.1097/NNA.0b013e31822edd79

PILONIETA, G. (2017). “Innovación disruptiva. Esperanza para la educación de futuro”. Educación y ciudad, (32), p. 53-64.

SHINGLER-NACE, A. (2020). COVID-19: When Leadership Calls. Nurse Leader. 18(3): p. 202-203. doi.org/10.1016/j.mnl.2020.03.017

SMOLA, B. (1988). Refinement and validation of a tool measuring leadership characteristcs of a baccalaureate students, in: Strickland, L., Waltz, C. (Eds.), Measurement of Nursing Outcomes. Spring Publishing Company, New York, NY, pp. 314-336.

WARD, S.L., LAFRAMBOISE, L.M., COSIMANO, A.J. (2016). Collaborative Student Leadership Conference. Journal of Professional Nursing. $\mathrm{N}^{\circ} \quad 32, \quad$ p. $\quad$ S63-S67. https://doi.org/10.1016/j.profnurs.2016.01.013 